\title{
Impact of endometrial microbiome on fertility
}

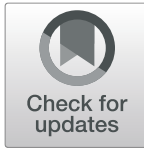

\author{
Aboubakr Mohamed ElnashariD
}

\begin{abstract}
Background: Next-generation sequencing isolates culturable and unculturable bacteria from the female reproductive tract. Current literatures surrounding the impact of endometrial microbiome on fertility are reviewed.

Main body: An abnormal endometrial microbiota has been associated with implantation failure pregnancy loss and other gynecological and obstetrical conditions. Identification of endometrial dysbiosis as a new cause of infertility opens a new microbiological field in the evaluation of endometrial factor, highlighting the relevance of assessing the uterine microbiota in infertile patients to restore a favorable endometrial flora in those patients with altered uterine microbiota to improve and personalize the clinical care of infertile patients. Understanding the significance of microbiome in the endometrium may completely change the therapeutic approach in the treatment of this part of the reproductive tract.
\end{abstract}

Conclusion: Investigation of the endometrial microbiota may be a future tool for improving reproductive outcomes in infertile patients. Further well-designed studies are required to establish its role in the evaluation and treatment of infertile patients

\section{Backgound}

\section{The reproductive tract microbiome}

\section{Definitions}

Microbiota: The microbial taxa that are associated with an environment and are revealed using molecular techniques such as $16 \mathrm{~S}$ rRNA sequencing [1].

Microbiome: Refers to the habitat as a whole, thus incorporating the biotic and abiotic factors, encompassing host and microorganism genomes and environmental conditions.

Dysbiosis: Qualitative and quantitative changes, their metabolic activity, and their local distribution.

Prebiotic: A non-digestible food ingredient that benefits the host by selectively stimulating the growth and/or activity of one or a limited number of bacteria in the colon, and thus improves host health.

Probiotic: Live microorganisms that when administered in adequate amounts confer a health benefit on the host.

\section{Correspondence: Elnashar53@hotmail.com}

Benha University Hospital, Banha, Egypt

\section{Methods of detection \\ Culture-based technologies}

Many microorganisms are not readily detected. As many as $50 \%$ of pathogens classified as "dominant" and $85 \%$ of "major" pathogens in wound infections are not identified by standard culture techniques [2]. While still foundational and often informative, it must be interpreted cautiously. Classical microbial culture provides a limited and biased view of the uterine environment, as not every bacterium is able to grow in standard laboratory conditions.

\section{New investigative techniques}

It includes DNA fingerprinting, microarrays, and targeted or whole genome sequencing. It depends on $16 \mathrm{~S}$ rRNA gene that is unique to bacteria and contains a number of hypervariable regions that serve as unique identifiers for a genus or species of bacterium. 16S rDNA gene sequencing is a reference method for bacterial identification and a standard method in bacterial community profiling. Sites in the body that are historically thought to be sterile, such as the uterine cavity and 
the placenta, are in fact colonized with their own unique microbiome [3]. For this reason, molecular technologies that are able to provide a 360 view of the uterine microbiota have been used to unravel the composition and impact of endometrial flora in patients subjected to assisted reproductive technologies.

Limitations of microbiome sequencing While sequencing can give insight into the makeup of the microbiome, it does not give information about its biologic function, such as antibiotic susceptibility testing [4].

\section{Characterization of the reproductive tract microbiome}

There is a continuum of slightly different microbiota expanding gradually from the vagina to the ovaries [5]:

1. The lower third of the vagina and posterior fornix: dominated by Lactobacillus spp. (99.99\%)

2. Cervical canal: Lactobacillus spp. (97.56\%)

3. Endometrium: Lactobacillus spp. forms a large portion of endometrial microbiome (30.6\%). Acinetobacter (9.07\%), Pseudomonas (9.09\%), Sphingobium (5\%), and Vagococcus (7.29\%)

4. At the openings of the fallopian tubes: the proportion of these bacteria increases while the median relative amount of Lactobacillus spp. is around $1.69 \%$

5. Peritoneal fluid from the pouch of Douglas: contains little to no Lactobacillus genus

\section{Vaginal microbiome}

In healthy women is generally dominated by Lactobacilli species [6]. Variation due to age and hormonal milieu is evident [7]. Vaginal flora has been classified into 5 community state types (CSTs): $\geq 70 \%$ of women demonstrated vaginal microbiota dominated by $L$. crispatus, $L$. gasseri, L. iners, or L. jensenii, corresponding to CST-I, CST-II, CST-III, and CST-V. A smaller proportion of women exhibit CST-IV, characterized by a lower percentage of Lactobacilli and dominance of anaerobic bacteria including Aerococcus, Atopobium, Dialister, Gardnerella, Megasphaera, Prevotella, and Sneathia.

\section{Endometrial microbiome}

For almost a century, gynecologists and scientists were convinced that a healthy uterus is sterile. Culture-based technology at the time of hysterectomy or targeted PCR identification for specific species showed the presence of both Lactobacillus as well as non-Lactobacillus species [8]. Like the normal vaginal microbiota, the endometrium of healthy and asymptomatic women is often dominated by Lactobacilli [9]. The upper genital tract microbiome is quantitatively and qualitatively different from that of the lower genital tract [8]. These discrepancies between the endometrial and vaginal microbiota rationalize the importance of analyzing the uterine microbiota in infertile patients as this is the maternal environment in which the embryo must adhere, implant, and grow to develop a healthy pregnancy to term. Interestingly, in up to $20 \%$ of subjects, significant differences between the bacterial composition of vaginal and endometrial samples have been observed, pointing to the analysis of the uterine cavity as the most straightforward way to predict implantation [10]. An abnormal endometrial microbiota has been associated with implantation failure, pregnancy loss, and gynecological and obstetrical conditions.

\section{Challenges of analyzing the endometrial microbiota}

The vaginal microbiota have been estimated to harbor approximately 1010-1011 bacteria, while the endometrium may contain four orders of magnitude fewer bacteria than the vagina, which is considered a low-biomass microbiome [11]. Low-biomass microbiomes (endometrial, urine, blood) may play important roles in microbial homeostasis and physiology, but their study is often biased by potential contaminations coming from bacteria or bacterial DNA present in the air, the laboratory equipment, and reagents that are inadvertently incorporated into the samples during sample collection, processing, and analysis. For this reason, it is of outstanding relevance that researchers working with low-biomass samples follow strict protocols to avoid misleading conclusions about the sequencing results. These measures would include the prevention of contamination before sequencing by maximizing the protective equipment, during sequencing by including sufficient negative and blank controls, and after sequencing by developing bioinformatic pipelines to track and subtract potential contaminants from the bonafide microbiome coming from the sample [12].

\section{Main text \\ Endometrial microbiome and infertility}

The role of endometrial microbiota at the embryomaternal interface in the onset of pregnancy is of great interest in reproductive medicine, and a better understanding of what a healthy uterine environment is, and how to achieve it, would benefit not only women undergoing IVF but also every woman wishing to conceive [5]. Several publications support the theory that alterations in the endometrial microbiome may also impact the reproductive potential of infertile patients and perhaps, correcting microbial dysbiosis would lead to improve success [13]. Uterine infection is a known risk factor for infertility, as this pathogenic environment may entail inflammation and immune activation in the endometrium, 
impairing embryo implantation and the onset of a successful pregnancy. From the clinical point of view, the identification of endometrial dysbiosis as a new cause of infertility opens a new microbiological field in the evaluation of endometrial factor, highlighting the relevance of assessing the uterine microbiota in infertile patients to restore a favorable endometrial flora in those patients with altered uterine microbiota to improve and personalize the clinical care of infertile patients. The impact of endometrial microbiota on several causes of infertility is being investigated to improve clinical management of infertile patients with altered uterine microbiota.

\section{Endometriosis}

Several investigations have linked the presence of endometrial pathogens to endometriosis, providing a potential role of bacterial pathogens in the onset of the disease. The evidences supporting this hypothesis come from the increased isolation of Actinomyces, Corynebacterium, Enterococcus, E. coli, Fusobacterium, Gardnerella, Prevotella, Propionibacterium, Staphylococcus, and Streptococcus to the detriment of Lactobacillus spp. in endometrial samples and menstrual blood of patients with endometriosis, while bacteria from the Staphylococcaceae and Streptococcaceae families have been identified at the molecular level in ovarian endometrioma fluid [14]. Recently, the description of the reproductive tract microbiota by next-generation sequencing (NGS) has showed that patients with and without endometriosis-related infertility present a different microbiome [15]. This supports the correlation of endometriosis with the presence of endometrial infections that may impair contractility of the uterus, facilitating the retrograde seeding of endometrial cells [16]. A recently published study analyzing endometriotic lesions found that the microbial diversity of lesions was higher compared to eutopic endometrium, where Lactobacillus, Enterococcus, Gardnerella, Pseudomonas, Alishewanella, Ureaplasma, and Aerococcus prevailed [17].

\section{Adenomyosis}

Patients with adenomyosis present significant differences along their reproductive tract microbiome compared with subjects without the condition [18].

\section{Chronic endometritis}

Moreno et al. demonstrated, that molecular microbiology is a reliable, fast, and cheap diagnostic tool that allows for the detection of culturable and non-culturable bacteria associated with chronic endometritis and has $77 \%$ concordance with a combination of the classical diagnostic methods such as histology, hysteroscopy, and microbial culture [19]. This is very important information, as chronic endometritis can be asymptomatic, and is found in about $40 \%$ of infertile patients, likely causing repeated implantation failure or even recurrent miscarriage. The unequivocal diagnosis of chronic endometritis in infertile patients, using objective and reliable methods, could help to improve the clinical management of asymptomatic patients in whom chronic endometritis is not suspected or diagnosed.

\section{Endometrial microbiome in art}

Many studies showed that endometrial microbiome might influence endometrial receptivity.

\section{Using culture-based methods}

1. During the 1990s and 2000s, the association of endometrial infection with reproductive failure of IVF treatments was reported by several groups assessing the endometrial flora at the time of embryo transfer (ET) by microbial culture of the distal tip of the transfer catheter. In all these studies, the reproductive outcome was consistently poor upon isolation of endometrial pathogens as Streptococcus spp., Staphylococcus spp., Enterococcus spp., Escherichia coli, Klebsiella pneumoniae, and Gram-negative bacteria compared with cases with negative culture [8, 20-23].

2. On the other hand, isolation of Lactobacillus spp. was associated with increased implantation and pregnancy rates per transfer and lower miscarriage rates.

3. A comprehensive study showed the relevance of a healthy microbial environment at the window of implantation by culturing endometrial samples at the time of oocyte retrieval (when a prophylactic antibiotic was prescribed) and at ET, which was performed $48 \mathrm{~h}$ later [23]. In this study, patients with endometrial pathogens at the time of ET presented decreased clinical pregnancy rates per transfer (18.7\%) compared with those women with negative cultures or those who had responded to antibiotic therapy previous to ET ( $41.3 \%$ and $38.1 \%$, respectively), supporting the concept that reversibility of endometrial infections could improve reproductive outcomes in IVF patients

\section{Using 165 rRNA gene sequencing}

1. The endometrial microbiome of infertile patients with repeated implantation failure (RIF) or recurrent pregnancy loss (RPL) has been investigated, showing that the uterine microbiome of these patients is predominantly made of bacteria from the phyla Firmicutes, Bacteroidetes, and 
Proteobacteria [9]. These phyla contain several genera previously reported not only in the female reproductive tract, but specifically in endometrial samples: Firmicutes contains Lactobacilli, Streptococci, Staphylococci, and among others; Bacteroidetes contains Prevotella; and Proteobacteria contains Enterobacteria as E. Coli and $K$. pneumoniae.

2. Endometrial dysbiosis is a cause of implantation failure and pregnancy loss [21]. The endometrial microbiota are made of 108 components, with Lactobacillus spp. being the most abundant bacteria. Patients were classified as Lactobacillus dominated (LD) or non-Lactobacillus dominated (NLD ) with a cutoff value of Lactobacillus relative abundance over $90 \%$; this cutoff served as a significant variable able to predict reproductive success. NLD group, in comparison with the LD group, had significantly lower implantation $(23.1 \%$ vs $60.7 \%, p=0.02)$, pregnancy ( $33.3 \%$ vs $70.6 \%, p=$ 0.03 ), ongoing pregnancy ( $13.3 \%$ vs $58.8 \%, p=$ $0.02)$, and live birth $(6.7 \%$ vs $58.8 \%, p=0.002)$ rates. High levels of Lactobacillus (over $90 \%$ as defined by the group) are significantly associated with growing reproductive success in IVF.

3. Hormones and endometrial microbiota: Hormonal fluctuations, especially in estrogens, are implicated in the regulation of the vaginal microbiota and in the preparation of the endometrium for implantation and pregnancy [24]. It can be expected that hormones also influence the endometrial microbiota. Surprisingly, however, Moreno et al. [11] found that the endometrial microbiota does not change under hormonal influence in the period preceding implantation. These results suggest that we should start to consider the endometrial microbiota and its health state prior to beginning IVF, in order to maximize the chance of positive outcomes.

4. Effect of controlled ovarian stimulation (COS) and progesterone $(\mathrm{P})$ luteal supplementation on microbiota of women undergoing in vitro fertilization was studied [25]. Lactobacillus was the most prevalent genus in the vaginal samples, although its relative proportion was reduced by COS plus $P$ supplementation ( $71.5 \pm 40.6 \%$ vs. 61.1 $\pm 44.2 \%)$. In the vagina, an increase in pathogenic species was observed, involving Prevotella (3.5 \pm $8.9 \%$ vs. $12.0 \pm 19.4 \%$ ), and Escherichia coli-Shigella spp. $(1.4 \pm 5.6 \%$ vs. $2.0 \pm 7.8 \%)$. In the endometrium, the proportion of Lactobacilli slightly decreased $(27.4 \pm 34.5 \%$ vs. $25.0 \pm 29.9 \%)$; differently, both Prevotella and Atopobium increased $(3.4 \pm 9.5 \%$ vs. $4.7 \pm 7.4 \%$ and $0.7 \pm 1.5 \%$ vs. $5.8 \pm 12.0 \%)$. In both sites, biodiversity was greater after COS, particularly in the endometrial microbiota. COS and P supplementation significantly change the composition of vaginal and endometrial microbiota. The greater instability could affect both endometrial receptivity and placentation.

\section{Current treatments in clinical practice}

In the clinical setting, there is a demand to detect and to improve endometrial dysfunctions in order to treat uterine dysbiosis and to enhance infertility treatment outcomes [26]. However, there is no unified protocol for assessing the endometrial microbial composition, neither for the treatment of uterine dysbiosis. Pioneering studies based on NGS approaches already developed commercially available tests for assessing endometrial microbiome: the EMMA test by iGenomix $^{\circ}$ [27] and Endometrial Microbiome Test by Varinos Inc. [28]. The EMMA test is based on the previous study findings where Lactobacillus was dominating in the uterus and that Lactobacillus dominance correlated with reduced miscarriage and implantation failures and thus improved pregnancy rates in women undergoing IVF [10]. EMMA test classifies endometrial samples into Lactobacillus dominant and non-Lactobacillus dominant profiles. Once the sample is classified as non-Lactobacillus dominant, adequate treatment including antibiotics, probiotics, and prebiotics could be applied. In the same line, the Varinos test supports the Lactobacillus dominance in the uterus and categorizes endometrial microbiome as Lactobacillus dominant and non-Lactobacillus dominant [29]. Then, choices for intervention are suggested, such as uterine lavage for microbial eradication, eradication treatment with antibiotics, and/or taking probiotics and prebiotics for improving the microbiota. Undoubtedly useful, these tests are based on mostly observational studies and include a limited number of patients, resulting in rather limited evidence for testing and subsequent clinical decision-making in a clinical setting [30]. Clearly, more research in the field is required starting with the establishment of the core uterine microbiome before any treatment recommendations for "dysbiosis" are offered for patients.

The effects of antibiotics and probiotics supplementation on the microbiome of the genital tract

Antibiotics has been widely studied to treat BV and prevent preterm birth [31]. But the usefulness of antibiotic use before embryo transfer remains controversial; while it is efficient in reducing upper genital tract contamination, no beneficial role has been observed in pregnancy outcome. This can be due to the lack of specificity of broad-spectrum antibiotics that could impair not only 
the growth of dysbiotic bacteria, but also the protective Lactobacilli. Antibiotic therapy against CE improved implantation, clinical pregnancy, ongoing pregnancy, and live birth rates in a subsequent IVF cycle after CE was resolved. Treatment of CE in RIF patients before embryo transfer could be a useful intervention to eliminate the source of infection, improve the endometrial microbial health, and increase the live birth rates in these patients [32]. Antibiotic therapy has been also effective in patients with $\mathrm{CE}$ and unexplained infertility to increase their chances to conceive spontaneously and maintain a safe pregnancy to term after CE resolution.

Another possible strategy to modulate the reproductive tract microbiome is the use of probiotics. Several oral and vaginal probiotics are available, the majority of them including L. crispatus, L. gasseri, Lactobacillus plantarum, Lactobacillus reuteri, and Lactobacillus rhamnosus [33]. This could offer an interesting approach to restore a healthy microbiota while overcoming the disadvantages of antibiotic treatment such as antibiotic resistance, high rate of recurrent infections after treatment, and side effects derived from the clearance of endogenous off-target flora in other body sites. However, the efficacy of a probiotic therapy alone in reverting BV and other reproductive tract infections is not certain. For example, treatment with vaginal $L$. crispatus for one cycle results in colonization with this strain in up to $60 \%$ of patients. A two-step therapy with vaginal probiotics following antibiotic treatment could be useful to first fight the fastidious bacteria and then repopulate the reproductive tract with Lactobacillus strains [34].

\section{Conclusions}

The existence of a reproductive microbiota continuum has highlighted the importance of a healthy microbiome in all the steps of reproduction. Next-generation sequencing isolates culturable and unculturable bacteria from the female reproductive tract. Endometrial microbiome is becoming better characterized and its importance in gynecologic and reproductive health is increasing. Low biomass microbiome has an increasing importance in today's fertility studies. An abnormal endometrial microbiota has been associated with implantation failure pregnancy loss and other gynecological and obstetrical conditions. Understanding the significance of microbiome in the endometrium may add value to the current therapeutic approach in the treatment of this part of the reproductive tract. More research is needed to describe and understand the role of the endometrial microbiome in endometrial receptivity and the outcome of in vitro fertilization.

\section{Abbreviations}

CSTs: Community state types; PCR: Polymerase chain reaction; NGS: Nextgeneration sequencing; ET: Embryo transfer; RIF: Repeated implantation failure; RPL: Recurrent pregnancy loss; LD: Lactobacillus dominated; NLD: Non-Lactobacillus dominated; IVF: In vitro fertilization; COS: Controlled ovarian stimulation; P: Progesterone; EMMA: Endometrial Microbiome Metagenomic Analysis; CE: Chronic endometritis; BV: Bacterial vaginosis

\section{Acknowledgements}

None

Author's contributions

The author read and approved the final manuscript.

Funding

None

Availability of data and materials

Not applicable

Ethics approval and consent to participate

Not applicable

Consent for publication

Yes

\section{Competing interests}

None

Received: 26 August 2020 Accepted: 27 December 2020

Published online: 13 January 2021

\section{References}

1. Cho I, Blaser MJ (2012) The human microbiome: at the interface of health and disease. Nat Rev Genet. 13:260-270

2. Rhoads DD, Cox SB, Rees EJ, Sun Y, Wolcott RD (2012) Clinical identification of bacteria in human chronic wound infections: culturing vs. 165 ribosomal DNA sequencing. BMC Infect Dis 12:321-325

3. Franasiak JM, Scott RT (2015) Reproductive tract microbiome in assisted reproductive technologies. Fertil Steril 104:1364-1371

4. Franasiak JM, Werner MD, Juneau CR, Tao X, Landis J, Zhan Y et al (2016) Endometrial microbiome at the time of embryo transfer: next-generation sequencing of the $16 \mathrm{~S}$ ribosomal subunit. J Assist Reprod Genet 33:129-136

5. Moreno I, Franasiak J (2017) Endometrial microbiota—new player in town. Fertil Steril 108:32-39

6. Moreno I, Simon C (2018) Relevance of assessing the uterine microbiota in infertility. Fertil Steril. 110:337-343

7. Moore E, Soules R, Klein A, Fujimoto Y, Agnew J, Eschenbach A (2000) Bacteria in the transfer catheter tip influence the live-birth rate after in vitro fertilization. Fertil Steril 74:1118-1124

8. Salim R, Ben-Shlomo I, Colodner R, Keness Y, Shalev E (2002) Bacterial colonization of the uterine cervix and success rate in assisted reproduction: results of a prospective survey. Hum Reprod 17:337-340

9. Verstraelen $H$, Vilchez-Vargas R, Desimpel F, Jauregui R, Vankeirsbilck N, Weyers S et al (2016) Characterisation of the human uterine microbiome in non-pregnant women through deep sequencing of the $\mathrm{V} 1-2$ region of the 165 rRNA gene. Peer J 4:e1602

10. Franasiak M, Werner D, Juneau $R$, Tao X, Landis J, Zhan $Y$ et al (2016) Endometrial microbiome at the time of embryo transfer: next-generation sequencing of the $16 \mathrm{~S}$ ribosomal subunit. J Assist Reprod Genet 33:129-136

11. Moreno I, FM C n, Vilella F, Valbuena D, Martinez-Blanch JF, JimenezAlmazan J et al (2016) Evidence that the endometrial microbiota has an effect on implantation success or failure. Am J Obstet Gynecol 15:684-703

12. Mitchell M, Haick A, Nkwopara E, Garcia R, Rendi M, Agnew K et al (2015) Colonization of the upper genital tract by vaginal bacterial species in nonpregnant women. Am J Obstet Gynecol 212(611):e1-e9

13. Weiss S, Amir A, Hyde R, Metcalf L, Song J, Knight R (2014) Tracking down the sources of experimental contamination in microbiome studies. Genome Biol 15:564-569

14. Franasiak JM, Scott RT (2015) Introduction: Microbiome in human reproduction. Fertil Steril. 104:1341-1343

15. Khan N, Fujishita A, Kitajima M, Hiraki K, Nakashima M, Masuzaki H (2014) Intrauterine microbial colonization and occurrence of endometritis in women with endometriosis. Hum Reprod 29:2446-2456 
16. Chen C, Song X, Wei W, Zhong H, Dai J, Lan Z et al (2017) The microbiota continuum along the female reproductive tract and its relation to uterinerelated diseases. Nat Commun 8:875-879

17. Pinto V, Matteo M, Tinelli R, Mitola PC, De Ziegler D, Cicinelli E (2015) Altered uterine contractility in women with chronic endometritis. Fertil Steril 103:1049-1052

18. Hernandes C, Silveira P, Rodrigues Sereia F, Christo P, Mendes H, Valter de Oliveira F, Podgaec S (2020) Microbiome profile of deep endometriosis patients: comparison of vaginal fluid, endometrium and lesion. Diagnostics 10:163-168

19. Gajer P, Brotman RM, Guoyun B et al (2012) Temporal dynamics of the human vaginal microbiota. Sci Transl Med. 4:132-152

20. Fanchin R, Harmas A, Benaoudia F, Lundkvist U, Olivennes F, Frydman R (1998) Microbial flora of the cervix assessed at the time of embryo transfer adversely affects in vitro fertilization outcome. Fertil Steril 70:866-870

21. Moore DE, Soules MR, Klein NA, Fujimoto VY, Agnew KJ, Eschenbach DA (2000) Bacteria in the transfer catheter tip influence the live-birth rate after in vitro fertilization. Fertil Steril 74:1118-1124

22. Selman H, Mariani M, Barnocchi N, Mencacci A, Bistoni F, Arena S et al (2007) Examination of bacterial contamination at the time of embryo transfer, and its impact on the IVF/pregnancy outcome. J Assist Reprod Genet 24:395-399

23. Egbase E, Udo E, al-Sharhan M, Grudzinskas G (1999) Prophylactic antibiotics and endocervical microbial inoculation of the endometrium at embryo transfer. Lancet 354:651-652

24. Wessels JM, Felker AM, Dupont HA, Kaushic C (2018) The relationship between sex hormones, the vaginal microbiome and immunity in HIV-1 susceptibility in women. Dis Model Mech. 11

25. Carosso A, Revelli A, Gennarelli G, Canosa S, Cosma S, Borella F, Tancredi A, Paschero C, Boatti L, Zanotto E, Sidoti F, Bottino P, Costa C, Cavallo R, Benedetto C (2020) Controlled ovarian stimulation and progesterone supplementation affect vaginal and endometrial microbiota in IVF cycles: a pilot study. J Assist Reprod Genet. 10:815-820

26. Molina N, Sola-Leyva A, Saez-Lara M, Plaza-Diaz J, Tubic-Pavlovic A, Romero B, Clavero A, Mozas-Moreno J, Fontes J, Altmäe S (2020) New opportunities for endometrial health by modifying uterine microbial composition:present or future? Biomolecules 10:593-611

27. Igenomix Foundation. EMMA Endometrial Microbiome Metagenomic Analysis: a screening test to evaluate the endometrium at the microbiological level. Available online: https://www.igenomix.com/geneticsolutions/ emma-clinics/ (accessed on 23 August 2020).

28. Varinos Inc. Innovate Reproductive Health by Genomic Testing. Available online: https://www.varinos.com/english (accessed on 23 August 2020).

29. Kyono K, Hashimoto T, Kikuchi S, Nagai Y, Sakuraba Y (2019) A pilot study and case reports on endometrial microbiota and pregnancy outcome: An analysis using $16 \mathrm{~S}$ rRNA gene sequencing among IVF patients, and trial therapeutic intervention for dysbiotic endometrium. Reprod Med Biol. 18: 72-82

30. Haahr T.; Jensen S; Humaidan P. Research and business - the yin and yang in modern medicine. Reprod Biomed. Online 2020; 40:613-616.

31. Kroon SJ, Ravel J, Huston WM (2018) Cervicovaginal microbiota, women's health, and reproductive outcomes. Fertil Steril. 110(3):327-336

32. Vitagliano A, Saccardi C, Noventa M et al (2018) Effects of chronic endometritis therapy on in vitro fertilization outcome in women with repeated implantation failure: a systematic review and meta-analysis. Fertil Steril. 110(1):103-112.e1

33. Sun X, Fiala JL, Lowery D (2016) Patent watch: Modulating the human microbiome with live biotherapeutic products: intellectual property landscape. Nat Rev Drug Discov. 15:224-225

34. Bradshaw CS, Pirotta M, De Guingand D et al (2012) Efficacy of oral metronidazole with vaginal clindamycin or vaginal probiotic for bacterial vaginosis: randomised placebo-controlled double-blind trial. PLoS One. 7: e34540

\section{Publisher's Note}

Springer Nature remains neutral with regard to jurisdictional claims in published maps and institutional affiliations.

\section{Submit your manuscript to a SpringerOpen ${ }^{\circ}$ journal and benefit from:}

- Convenient online submission

- Rigorous peer review

- Open access: articles freely available online

- High visibility within the field

- Retaining the copyright to your article

Submit your next manuscript at $\boldsymbol{\nabla}$ springeropen.com 